\title{
Nuevos hospedadores para las garrapatas Amblyomma tigrinum y Rhipicephalus sanguineus (Acari: Ixodidae) en Chile
}

\author{
New hosts for the ticks Amblyomma tigrinum and Rhipicephalus sanguineus \\ (Acari: Ixodidae) in Chile \\ D González-Acuña ${ }^{1 *}$, G Valenzuela ${ }^{1}$, L Moreno ${ }^{1}$, K Ardiles ${ }^{1}$, A Guglielmone ${ }^{2}$ \\ ${ }^{1}$ Facultad de Medicina Veterinaria, Universidad de Concepción, Chillán, Chile. \\ 2 Instituto Nacional de Tecnología Agropecuaria, CC 22, CP 2300 Rafaela, Santa Fe, Argentina.
}

\begin{abstract}
SUMMARY
For the first time in Chile, cases of nymphs of the brown dog tick (Rhipicephalus sanguineus) being found on two goats (Capra hircus), and adult (one male and a gravid female) white stripped ticks (Amblyomma tigrinum) being found on cattle (Bos taurus) have been reported. The aim of this review is to discuss the importance of these findings.
\end{abstract}

Palabras clave: Rhipicephalus sanguineus, Amblyomma tigrinum, garrapatas, Chile

Key words: Rhipicephalus sanguineus, Amblyomma tigrinum, ticks, Chile.

\section{INTRODUCCION}

La garrapata café del perro, Rhipicephalus sanguineus (Latreille 1806), tiene su origen en África desde donde se dispersó a través de todo el mundo. Su distribución es probablemente la más amplia a nivel mundial, se encuentra entre los $45^{\circ}$ latitud norte y $45^{\circ}$ latitud sur (Keirans 1992). En Chile se describió por primera vez en al año 1976 en un perro en Santiago (Región Metropolitana) y desde entonces se ha diseminado rápidamente debido a su alto poder biótico, a las excelentes condiciones ecológicas para su desarrollo y, además, a que no fue controlada oportunamente (Alcaíno 1985).

En las regiones tropicales y subtropicales, esta especie puede ser encontrada durante todo el año (Strickland y col 1976); sin embargo, en países con cuatro estaciones bien definidas son más abundantes en primavera y verano (Alcaíno y col 1990), comenzando a disminuir paulatinamente en verano y otoño (Alcaíno 1985). En el neotrópico es considerada el ectoparásito de mayor importancia en los perros (Guglielmone y col 2003). En Chile se ha registrado en perros y menos comúnmente en gatos en el centro y centro-sur de Chile (Alcaíno 1985, Alcaíno y col 1990, Schenone 1996, Morales y col 2001, Muñoz y Casanueva 2001) como también en hospedadores inusuales como el guarén

Aceptado: 11.04.2006.

* Facultad de Medicina Veterinaria, Universidad de Concepción, Casilla 537, Fax: 42-270212, Chillán, Chile. E-mail: danigonz@udec.cl
(Rattus norvegicus), el conejo doméstico (GonzálezAcuña y col 2003 ${ }^{\text {b; }}$ González-Acuña 2004) y el hombre (Schenone 1996).

Por otro lado, Amblyomma tigrinum Koch, 1844 es una especie sudamericana descrita en Argentina, Bolivia, Brasil, Guyana Francesa, Paraguay, Perú, Uruguay, Venezuela y Chile (Jones y col 1972). Su primera descripción en Chile fue hecha por Tagle y Alvarez en 1957 como A. maculatum en zorros (Pseudalopex spp.) provenientes de Arauco, pero Tagle y Alvarez (1959) revalidan su diagnóstico como A. tigrinum. Más tarde, Tagle (1971) describe ejemplares adultos de A. tigrinum en perros y zorros de Nahuelbuta y Valparaíso y recientemente fueron descritos en perros en la ciudad de Concepción (Muñoz y Casanueva 2002) y también en zorros de San Antonio (González-Acuña y col 2003ª). En Ñuble se registraron los estados larvarios y de ninfa de A. tigrinum en las aves silvestres codorniz (Callipepla califronica), tórtola (Zenaida auriculata) y perdiz (Nothoprocta perdicaria) (González-Acuña y col 2004).

El presente estudio presenta los primeros registros en Chile de $R$. sanguineus y A. tigrinum en cabra y bovino respectivamente.

\section{MATERIAL Y METODOS}

La recolección de los Ixodidos fue realizada el día 3 y 14 de noviembre de 2004 en forma manual de los hospedadores Capra hircus y Bos taurus respectivamente. Las especies llegaron como pacientes a la clínica de la Facultad de Medicina Veterinaria de la Universidad de Concepción (36³6’S; 7303'W). Las cabras (madre e 
hija) provenían de San Ignacio $\left(37^{\circ} 13^{\prime} S ; 72^{\circ} 31^{\prime} \mathrm{W}\right)$ y fueron traídas debido a debilidad postparto. De ambos ejemplares fueron aislados en total 54 ninfas de garrapatas que se ubicaban ingurgitadas preferencialmente en la zona de la tabla del cuello. El bovino correspondió a un ternero macho proveniente de la localidad de Quirihue $\left(36^{\circ} 17^{\prime} \mathrm{S} ; 72^{\circ} 32^{\prime} \mathrm{W}\right)$ que fue traído debido a una severa hipovitaminosis A. Desde este ejemplar se aisló un macho y una hembra ingurgitada en el dorso del animal, la cual fue mantenida bajo condiciones controladas de temperatura y humedad $\left(24^{\circ} \mathrm{C} \mathrm{y} 80 \% \mathrm{H}^{\circ}\right)$ oviponiendo 10 días después de la extracción aproximadamente 4.000 huevos. Los ejemplares de garrapatas colectados fueron conservados en alcohol $70 \%$ y determinados de acuerdo con las descripciones de Boero (1957) y Estrada-Peña y col (2005). El material estudiado fue depositado en la colección del laboratorio de Zoología de la Facultad de Medicina Veterinaria de la Universidad de Concepción.

\section{RESULTADOS Y DISCUSION}

Las 54 ninfas aisladas de cabra fueron identificadas como $R$. sanguineus. A pesar que los principales hospedadores de $R$. sanguineus son carnívoros (Guglielmone y col 2003) existen varios diagnósticos en otros tipos de hospedadores en Chile (mencionados en la introducción) y en otros países neotropicales como conejos silvestres (Ivancovich y Luciani 1992), marsupiales (Santa Cruz y col 1998), palomas (Diogo y col 2003), bovinos (IICA 1988), tamandua (Floch y Fauran 1958), reptiles como aligator y tortugas (Keirans 1985) entre otros.

En el neotrópico, hasta la fecha existen tres registros de parasitismo por $R$. sanguineus en cabra que corresponden a Van Volkenberg (1939), en Mayaguez (Puerto Rico), Barrera (1956) en la localidad de Torreón en México e Ivancovich y Luciani (1992) en Colonia Benítez (Argentina). La garrapata café del perro se describió en Chile hace menos de 30 años y debido a que la cabra no ha sido un hospedador regular para ixodoideos (GonzálezAcuña y Guglielmone 2005), la adaptación de $R$. sanguineus a la cabra y también otros animales domésticos podría llegar a jugar un importante rol en el mantenimiento de la especie. El presente registro es el primero para Chile de un Ixodideo en cabra y, además, es el primero en el que $R$. sanguineus parasita a un Artiodactyla en el país, por lo que sería de importancia considerar los perros como posibles fuentes de contagio para los caprinos y otros animales y, a su vez, estos jugar un papel como reservorios de la mencionada garrapata.

Las garrapatas aisladas del bovino fueron identificadas como un macho y una hembra grávida de A. tigrinum. Guglielmone y col 2003 describen A. tigrinum como una garrapata que parasita principalmente en carnívoros. Además, se ha descrito su hallazgo en el hombre (Floch y Fauran 1958, Ivancovich 1980, Evans y col 2000). En el neotrópico A. tigrinum ha sido registrado parasitando a bovinos en Argentina (Gugliemone y col 2000), en Paraguay (Massi Pallares y Benítez Usher 1982) y Uruguay (Sampaio y Larrosa 1992). La hembra de A. tigrinum ovipuso cerca de 4.000 huevos de los cuales 585 no eclosionaron.

En Chile, los registros de garrapatas en bovinos se restringen al trabajo de Descazeaux (1925), el que describió Ornithodoros megnini (=Otobius megnini) en el norte y centro de Chile. Además, Gervais (1849) reporta Ixodes ricinus en bovinos, sin embargo, se asume que I. ricinus es una especie paleártica y que el registro hecho por Gervasis podría tratarse de I. pararicinus (González-Acuña y Guglielmone 2005).

Sería de interés un estudio de abundancia de los ixódidos $R$. sanguineus y A. tigrinum en animales domésticos y correlacionar prevalencia con la presencia de perros parasitados en el área de estudio.

\section{RESUMEN}

Se registran, por primera vez en Chile, ninfas de garrapata café del perro (Rhipicephalus sanguineus) parasitando dos ejemplares de cabras (Capra hircus) y adultos (un macho y una hembra gravida) de la garrapata de listas blancas (Amblyomma tigrinum) parasitando a un bovino (Bos taurus). La importancia de los presentes reportes, hechos en la provincia de Nuble, son discutidos.

\section{REFERENCIAS}

Alcaíno H. 1985. Antecedentes sobre la garrapata café del perro (Rhipicephalus sanguineus). Monog Méd Vet 7, 48-55.

Alcaíno H, T Gorman, F Jiménez. 1990. Ecología del Rhipicephalus sanguineus (Ixodidae) en la Región Metropolitana de Chile. Arch Med Vet 22, 159-168.

Barrera A. 1956. Algunas consideraciones biológicas sobre Rhipicephalus sanguineus (Latreille), 1806, (sic) transmisor de fiebre manchada en el norte y noroeste de México. Rev Inst Salub Enf Trop 16, 51-59.

Boero JJ. 1957. Las garrapatas de la República Argentina (Acarina: Ixodoidea). Depto Edit Univ Buenos Aires, Buenos Aires, Pp 113.

Descazeaux MJ. 1925. Sur la presence au Chili de l'Ornithodoros megnini. Bull Soc Path Exot 18, 408-409.

Diogo AA, L Guerim, JR Pires, ALG Cuoto, NM Serra Freire. 2003. Parasitismo por Rhipicephalus sanguineus Latreille, $1806 \mathrm{em}$ Columba livia Linnaeus na cidade do Rio de Janeiro, Brasil. Entomol Vectores 10, 277-280.

Estrada-Peña A, JM Venzal, AJ Mangold, MM Cafrune, AA Guglielmone. 2005. The Amblyomma maculatum Koch, 1844 (Acari: Ixodidae: Amblyomminae) tick group: diagnostic characters, description of the larva of A. parvitarsum Neumann, 1901, 16S rDNA sequences, distribution and hosts. Syst Parasitol 60, 99-112.

Evans DE, JR Martins, AA Guglielmone. 2000. A review of the ticks (Acari: Ixodidae) of Brazil, their hosts and geographic distribution -1. The state of Rio Grande do Sul, Southern Brazil. Mem Inst Oswaldo Cruz 95, 453-470.

Floch H, P Fauran. 1958. Ixodidés de la Guyane et des Antilles Françaises. Arch Inst Pasteur Guyane 446, 1-94.

Gervais P. 1849. Arácnidos. En: Historia física y política de Chile. C Gay, París, Pp 5-52.

González-Acuña D, JM Venzal, JE Keirans, AA Guglielmone. 2003a. The genus Amblyomma Koch, 1844 (Acari: Ixodidae) in Chile, 
with new records of A. argentinae Neumann, 1904 and A. tigrinum Koch, 1844. Syst Appl Acarol 8, 85-88.

González-Acuña D, JM Venzal, AA Guglielmone. 2003b. Primer registro de Rhipicephalus sanguineus (Acarina: Ixodidae) en Rattus norvergicus (Mammalia: Rodentia) en Chile. Gayana Zool 67, 121-122.

González-Acuña D. 2004. El conejo (Oryctolagus cuniculus, Mammalia: Lagomorfo) como hospedador de la garrapata café del Perro (Rhipicephalus sanguineus, Acari: Ixodidae). Ciencia Ahora 7, 100-103.

González-Acuña D, J Venzal, A Guglielmone, A Daugschies, L Rubilar-Contreras, O Skewes-Ramm. 2004. First record of immature stages of Amblyomma tigrinum (Acari: Ixodidae) on wild birds in Chile. Exp Appl Acarol 33, 153-156.

González-Acuña D, AA Guglielmone. 2005. Ticks (Acari: Ixodoidea: Argasidae, Ixodidae) of Chile. Exp Appl Acarol 35, 147- 163.

Guglielmone AA, AJ Mangold, CE Luciani, AE Viñabal. 2000. Amblyomma tigrinum (Acari:Ixodidae) in relation to phytogeography of central-northern Argentina with note on hosts and seasonal distribution. Exp Appl Acarol 24, 983-900.

Guglielmone AA, A Estrada-Peña, JE Keirans, RG Robbins. 2003. Ticks (Acari: Ixodidae) of the Neotropical zoogeographic region. Int. Cons. Ticks Tick-Borne Dis., Atalanta, Houten, The Netherlands, Pp 174.

Instituto Interamericano de Cooperación para la Agricultura. 1988. Estudio ecológico y epidemiológico de garrapatas en Guatemala. Inf Coop Técn IICA-MAGA, Pp 43.

Ivancovich JC. 1980. Reclasificación de algunas especies de garrapatas del género Amblyomma (Ixodoidea) en Argentina. Rev Inv Agropec 15, 673-682.

Ivancovich J, C Luciani. 1992. Las garrapatas de Argentina. Monogr Asoc Arg Parasitol Vet, Pp 95.

Jones EK, CM Clifford, JE Keirans, GM Kohls. 1972. The ticks of Venezuela (Acarina: Ixodoidea) with a key to the species of Amblyomma in the Western Hemisphere. Brigham Young Univ. Sci Bull Biol Ser 17, 1-40.

Keirans JE. 1985. George Henry Falkiner Nuttall and the Nuttall tick catalogue. U S Dept Agric, Agric Res Ser Misc Pub (1438), Pp 1.785 .
Keirans JE. 1992. Systematics of the Ixodida (Argasidae, Ixodidae, Nuttalliellidae): an overview and some problems. En: Fivaz B, Petney T, Horak I (eds.). Tick vector biology, medical and veterinary aspects. Springer-Verlag, Berlin, Pp 1-21.

Massi Pallarés R, CA Benítez Usher. 1982. De la distribución de Ixodina (Vander Hammen, 1968) en el Paraguay. Rev Paraguaya Microbiol 17, 49-52.

Morales MA, L Ibarra, L Cáceres. 2001. Infestación por garrapatas en perros y viviendas del gran Santiago, Chile. Resúmenes del VII Congreso Nacional de Veterinaria, Montevideo, Uruguay, Pp 5.

Muñoz LE, ME Casanueva. 2001. Estado actual del conocimiento de las garrapatas (Acari: Ixodidae) asociadas a Canis familiaris L. Gayana 65, 193-210.

Muñoz LE, ME Casanueva. 2002. Garrapatas (Acari: Ixodidae) en perros de la ciudad de Concepción. Arch Med Vet 34, 131-134.

Sampaio I, A Larrosa. 1992. Hallazgo de Amblyomma tigrinum (Koch 1884) (sic) (Acari, Ixodidae) en el Uruguay. Bol Soc Zool Uruguay, 7, 1-87.

Santa Cruz AM, O Prieto, OJ Lombardero, L Gómez, N Schleiber. 1998. First finding of Rhipicephalus sanguineus Latreille, 1806 (Acari: Ixodidae) infesting Didelphis albiventris (Marsupialia: Didelphidae) in the province of Corrientes, Argentina. Resúmenes XIII Congreso Asociación Mundial Medicina Veterinaria de Pequeños Animales, Buenos Aires 2, 787.

Schenone H. 1996. Diagnósticos hechos a 1.384 pacientes que consultaron por probable mordedura de arañas o picaduras de insectos. Bol Chil Parasitol 51, 20-27.

Strickland RK, RR Gerrish, JL Hourrigan, GO Schubert. 1976. Ticks of Veterinary Importance. Agriculture Handbook $\mathrm{N}^{\circ} 485$. United States Department of Agriculture, Washington DC, Pp 122.

Tagle I. 1971. Ixodoidea en Chile. Bol Chil Parasitol 26, 46-49.

Tagle I, V Alvarez. 1957. Existencia de Amblyomma maculatum Koch 1844 en zorros de Chile. Bol Chil Parasitol 12, 66.

Tagle I, V Alvarez. 1959. Rectificación de diagnóstico: Amblyomma tigrinum, Koch en lugar de Amblyomma maculatum, Koch. Bol Chil Parasitol 14, 56-57.

Van Volkenberg HL. 1939. An annotated check list of the parasites of animals in Puerto Rico. Un. St. Dept. Agric. Circ. Puerto Rico Exp. Stn., Pp 12. 